\title{
Probiotic properties of an indigenous Pediococcus pentosaceus strain on Tenebrio molitor larval growth and survival
}

\author{
A. Lecocq ${ }^{1}{ }^{*}$, M.E. Natsopoulou ${ }^{1}$, I.E. Berggreen ${ }^{2}$, J. Eilenberg ${ }^{1}$, L.-H. Lau Heckmann ${ }^{2}$, H.V. Nielsen ${ }^{3}$, C.R. Stensvold ${ }^{3}$ \\ and A.B. Jensen ${ }^{1}$ \\ ${ }^{1}$ Department of Plant and Environmental Sciences, University of Copenhagen, Thorvaldsensvej 40, 1871 Frederiksberg \\ C, Denmark; ${ }^{2}$ Danish Technological Institute, Life Sciences, Kongsvang Allé 29, 8000 Aarhus, Denmark; ${ }^{3}$ Department of \\ Bacteria, Parasites E Fungi, Statens Serum Institut, Artillerivej 5, 2300 Copenhagen, Denmark; antoine@plen.ku.dk
}

Received: 27 November 2020 / Accepted: 7 January 2021

(C) 2021 Wageningen Academic Publishers

OPEN ACCESS CC)

\begin{abstract}
Optimising the production of insects for food and feed and ensuring their health are growing concerns for producers. Insects suffer from a range of insect pathogenic microorganisms, and the management of such diseases is essential. One solution is the introduction of beneficial probiotic bacteria into the diet of the insects. Here, we show that a lactic acid bacterial strain, Pediococcus pentosaceus, isolated from the gut of the mealworm, Tenebrio molitor, was able to inhibit the growth of selected insect pathogens in vitro. Using in vivo assessments of the host's fitness benefits conferred by the lactic bacterium we show a significant effect of $P$. pentosaceus on larval growth rate and survival into adulthood. Gut microbiota analysis focusing on bacterial composition based on 16S rRNA gene amplicon sequencing suggests that $P$. pentosaceus could have successfully colonised the guts, or altered their bacteria, of the larvae that received it. Finally, we discuss our results in the context of mass insect production systems and outline the remaining work needed to explore and secure the role of beneficial bacterial additives in the field.
\end{abstract}

Keywords: insect, production, food, feed, probiotic, bacteria, pathogen, microbiome

\section{Introduction}

With a growing global demand for animal protein (Pelletier and Tyedmers, 2010) and a need to reduce the footprint of livestock production on the planet (Carlsson-Kanyama, 1998; Foley et al., 2011), a number of insects species are being reared as alternative animal protein sources for food and feed (Stoops et al., 2016; Van Huis, 2013; Yen, 2009). Mass production of any animal comes with its own challenges. Among factors to consider is insect health, which influences mortality and growth rate and thus reduces insect production. One of the key species being produced is the mealworm, Tenebrio molitor (Coleoptera: Tenebrionidae). It is a popular insect already used for many years in research as a model organism. With a rich knowledge base in addition to containing a substantial source of protein, fat and essential amino acids (Ghaly and Alkoaik, 2009), T. molitor is a strong candidate to be used on a large scale, as food for humans or feed for livestock
(Van Huis, 2013). As a result, the production of T. molitor is rapidly growing. Larvae are reared in dense conditions and in great numbers, often in closed and automated production systems. Insect diseases from fungal, bacterial and nematode sources, among others, are known to affect this species (Eilenberg et al., 2015); yet, we are still lacking knowledge with regards to methods to avoid or manage such diseases (Lecocq et al., 2019).

Probiotics were first defined in 1965 as 'growth promoting factors produced by microorganisms.' (Lilly and Stillwell, 1965). In 2001, the Food and Agriculture Organization of the United Nations and the World Health Organization (FAO/WHO) referred to them as 'live microorganisms which when administered in adequate amounts confer a health benefit to the host', a definition which was reaffirmed by an expert panel convened in 2013 (Hill et al., 2014). A recent study showed that probiotics appear to provide some protection against pathogens in chickens (Islam and 
Yang, 2016). Furthermore, Grau et al. (2017b) demonstrated the potential for one probiotic isolate to act as a protective agent towards a bacterial disease in the model insect species Tribolium castaneum (Coleoptera: Tenebrionidae), both in vitro and in vivo although this isolate also had side effects such as a reduction in insect lifespan and reduced fertility (Grau et al., 2017b). The mechanisms behind the beneficial effects on vertebrate and invertebrate health are not fully understood but likely include modification of the gut microbiota, production of anti-microbial compounds, strengthening of the gut epithelial barrier and modulation of the immune system (Bermudez-Brito et al., 2012; Oelschlaeger, 2010). Probiotic effects might even be passed down to the final consumer, given the right processing conditions, keeping the microbiota of the insect intact (Grau et al., 2017a). While research evaluating the risk of pathogens in the production of insects destined for the food and feed industry has been going on for some time, alternatives to antibiotic use, as seen in many other livestock production systems, should be evaluated. Probiotic additives could be a solution, increasing insect health and facilitating the development of the mass-reared mealworms. The inclusion of probiotic bacteria as an additive in massreared mealworm diets should result in improved growth, survival and health in the insects, benefiting the industry.

In this study, focusing on lactic acid bacteria as the major representatives of probiotics (Holzapfel and Schillinger, 2002), we isolated and identified a strain of Pediococcus pentosaceus (Lactobacillales: Lactobacillaceae) from the gut of $T$. molitor larvae. We investigated its potential to influence the growth of Gram-positive and Gram-negative pathogenic bacteria in vitro. Subsequently, we used a formulation of the bacteria, which had the potential to be used as a powdered additive, to test the effect on larval growth, survival, and adult female egg-laying capacity in a mealworm production system. Our results, supported by $T$. molitor larval gut bacterial microbiota analysis, shed light on the changes brought about by the addition of $P$. pentosaceus to the insect's diet.

\section{Materials and methods}

\section{Sources of insects}

The T. molitor insects used in this experiment were sourced from the Danish Technological Institute (DTI) pilot production facility in Aarhus, Denmark. We reared the insects used for the isolation of bacteria at $30{ }^{\circ} \mathrm{C}$ and $60-70 \%$ relative humidity in darkness. For weight and size consistency, all larvae used for in vitro bacteria extractions and isolations were about $0.1 \pm 0.05 \mathrm{~g}$ per individual and 1-2 months old. For in vivo assays, we collected eggs from two separate cohorts of adult T. molitor from the same pilot production facility.

\section{Isolation of lactic acid bacteria from larval guts}

Bacteria were isolated from T. molitor using the following methods. Twenty 1-2-month-old larvae were randomly selected, isolated and starved individually in petri dishes for $18 \mathrm{~h}$. They were then externally sterilised with $75 \%$ ethanol for about $2 \mathrm{~min}$ and rinsed by dipping two times in sterilised water. We dissected the larvae following the method described by Wang and Zhang (2015). Briefly, a single insect was placed on a sterile surface, the posterior and anterior tips of the larvae were sliced with a scalpel, and the gut was pulled from the posterior end using sterilised forceps. A total of 20 guts were homogenised with sterilised plastic pestles, pooled together, and suspended in $10 \mathrm{ml}$ of water. All the work was done in a laminar flow cabinet.

Following dissection, we prepared serial dilutions ranging from $10^{-1}$ to $10^{-4}$ of the original $10 \mathrm{ml}$ suspension of T. molitor guts. We plated five replicates of each dilutions by spreading $100 \mu \mathrm{l}$ on De Man, Rogosa and Sharpe (MRS) agar ( $\mathrm{pH} 6.2 \pm 0.2$ ). The resulting 25 plates were incubated anaerobically in 2.51 Oxoid $^{\mathrm{Ts}}$ AnaeroJars (Thermo Fisher Scientific Inc., Waltham, MA, US) accompanied by AnaeroGen bags Thermo Fisher Scientific Inc.) and anaerobic indicators (to ensure redox conditions). Bacterial growth was allowed for $48 \mathrm{~h}$ at $31{ }^{\circ} \mathrm{C}$. Anaerobic incubation was selected in order to focus on gut colonising bacteria. We then selected 50 individual colonies, which appeared to differ in morphology and/or colour, and cultured them in liquid MRS broth for $24 \mathrm{~h}$ at $31^{\circ} \mathrm{C}$.

\section{Identification of bacterial isolates}

Bacterial DNA, from cells grown in liquid medium, was extracted using the CTAB DNA extraction protocol (Wilson, 2001). PCR reaction mix consisted of 0.5 $\mu \mathrm{M}$ of each $16 \mathrm{~S}$ RNA-genes-targeting primers (27F: 5'-AGAGT TTGATCMTGGCTCAG-3' and 806R: 5'-GGACTACNNGGGTATCTAAT-3'), $200 \mu \mathrm{M}$ of premixed dNTPs (Takara Bio, Shiga, Japan), 0.5U Taq polymerase (AmpliTaq Gold polymerase \& $\mathrm{MgCl}_{2}$, Applied Biosystems, ThermoFischer Scientific, Waltham, MA, USA), $1 \times$ PCR buffer and $1 \mu$ DNA template (ca $200 \mathrm{ng}$ ) to a final volume of $20 \mu \mathrm{l}$. The following thermal profile was used for amplification: $95^{\circ} \mathrm{C} / 3 \mathrm{~min} ; 33$ cycles at $94{ }^{\circ} \mathrm{C} / 90$ s, $50{ }^{\circ} \mathrm{C} / 35 \mathrm{~s}, 72{ }^{\circ} \mathrm{C} / 105 \mathrm{~s}$; and a final extension step at $72{ }^{\circ} \mathrm{C} / 3 \mathrm{~min}$. Prior to sequencing (Sanger sequencing, Genewiz, Takeley, UK) the PCR products were purified using a PCR purification kit (GE Healthcare, Chicago, IL, USA). For all sequences a BLAST search was carried out against the NCBI database using CLC Main Workbench 7 (Qiagen, Hilden, Germany). P. pentosaceus was the most abundant species and one isolate KVLB19-01 was selected for the rest of the experiment due to its undemanding growth requirements, including of temperature, $\mathrm{pH}$, and oxygen conditions (Zommiti et al., 2018). 


\section{In vitro antimicrobial activity}

The antimicrobial activity of the P. pentosaceus KVLB1901 was tested on four insect pathogenic bacterial species: Three strains of Bacillus thuringiensis (BT1236, BTUK and BT34; Novodor Biological control product provided by Neil Crickmore, University of Sussex, UK), Serratia marcescens KVLB18-05 (isolated from T. molitor), Serratia plymuthica KVLB18-06 (isolated from grasshoppers in Denmark) and Pseudomonas aeruginosa (isolated from Zophobas morio). We used the dual overlay technique (Magnusson and Schnürer, 2001) with slight modifications as described below. An overnight culture of P. pentosaceus grown in MRS at $33^{\circ} \mathrm{C}$ was cleaned and diluted in sterile water to a concentration of around $5 \times 10^{9} \mathrm{cfu} / \mathrm{ml}$ (optical density (OD) of 0.3 at $600 \mathrm{~nm}$ ). Ten microliters of the mixture was then spotted on a filter disc, itself placed on a MRS agar plate. As a control, the same volume of pure sterile water was also spotted on a filter disc. Petri dishes were incubated overnight in anaerobic conditions, in 2.5 l Oxoid AnaeroJars at $33{ }^{\circ} \mathrm{C}$. The next day, cultures of the selected bacterial pathogens grown in soft LB agar were mixed with $10 \mathrm{ml}$ soft agar $(0.8 \%)$ containing MRS and poured as an over layer on top of the incubated plates. Prior to mixing, the culture was adjusted to around $10^{8}$ cells $/ \mathrm{ml}$ (OD of $0.31-0.32$ at $600 \mathrm{~nm}$ ). All samples were once again incubated at $33^{\circ} \mathrm{C}$ for 24 hours in aerobic conditions. All the tests were replicated three times and inhibition of pathogenic bacteria was scored by the average of two transverse measurements of the radius of the inhibition zone. The final inhibition score (in $\mathrm{mm}$ ) was defined as the average from the measurements taken from three replicate petri dishes.

\section{Pediococcus pentosaceus cells obtention and treatments}

P. pentosaceus str. KVLB19-01 was grown in 500-ml vials containing MRS broth. After 24 hours incubation at $33^{\circ} \mathrm{C}$, bacterial cells were collected by centrifugation ( 15 minutes at 5,000 rpm), washed twice in phosphate buffer saline (PBS), transferred into 50-ml falcon tubes, washed and centrifuged once more, and re-suspended in $20 \mathrm{ml}$ PBS. All samples were then kept at $-80{ }^{\circ} \mathrm{C}$ until the time of the freeze-drying process. Freeze drying was carried out over 18 hours at $-60{ }^{\circ} \mathrm{C}$ in a Hetosicc CD52 freeze drier (Birkerød, Denmark). A portion of the resulting product was autoclaved at $121{ }^{\circ} \mathrm{C}$ for 15 min to kill the bacteria, and these were also used as dead control bacteria. A subset was then plated on MRS to confirm that no colonies grew after $24 \mathrm{~h}$.

\section{Diet preparation}

Diets were composed of a dry portion and a wet portion. The dry portion was a commercial feed developed by DTI for Adival (Billund, Denmark) composed of rape, rye and wheat and a mineral blend (dry matter: $85.74 \%$; crude protein: $14.83 \%$; carbohydrate: $81.05 \%$; fat: $1.75 \%)$. A total of $50 \mathrm{~g}$ of the commercial feed was administered at the beginning of the experiment. At week 4, all containers were cleaned and a new portion of $50 \mathrm{~g}$ of feed was administered. The wet portion of the diet consisted of dried and ground brewer's grain that was subsequently rehydrated by mixing grain and water (1:3). The brewer's grain was replaced every two days and varied in quantity, but was provided ad libitum, from $0.1 \mathrm{~g}$ at the beginning to $2 \mathrm{~g}$ every two days by the end of the experiment. Either live and autoclaved freeze dried bacteria were added to the dry portion of the diet. As with the dry food, bacteria were added at the beginning of the experiment and again at week 4 . Here, $0.5 \mathrm{~g}$ of freeze-dried material was mixed with the dry feed, resulting in $1 \times 10^{9} \mathrm{cfu} / \mathrm{g}$ for both live and autoclaved bacteria treatments.

\section{In vivo assays}

The potential probiotic effect of $P$. pentosaceus str. KVLB1901 was tested by measuring the development of T. molitor larvae with or without it. To distinguish between the effect of the bacteria as a live organism and their effect as a source of nutrients, another group ('autoclaved bacteria') was included in the study; instead of live bacteria, this group of larvae received dead (autoclaved) bacteria at the same concentration as the live bacteria. Eight replicates of one hundred, 24-hour-old larvae were set up for each of three treatments (i.e. Control, C; Bacteria, B; and Autoclaved bacteria, $A B$ ), resulting in 24 containers and 2,400 larvae at the beginning of the experiment. All containers were made of glass and had a volume of 0.61 .

We measured the weight of the larvae and their number in each replicate after $4,8,9$, and 10 weeks. We counted the number of adults (beetles) upon emergence and cumulated the numbers until week 20, at which time the last beetle emerged. Only intact, fully developed (six legs, full elytra) adults were counted. The effect of treatment on oviposition was evaluated by putting the first 20 adults emerging from each replicate into a separate container, fitted with a petri dish filled with flour and covered with a mesh and left for 7 days. The eggs laid by the beetles were collected at day 4 and day 7 and kept refrigerated before being counted. At the end of this experiment, all adults were sexed according to Bhattacharya et al. (1970) in order to determine the number of eggs laid per female.

\section{Bacterial microbiota sequencing and analysis}

When the larvae in a replicate reached an average weight of $0.12 \mathrm{~g} /$ larva (harvesting stage in production), the guts from two larvae per replicate per treatment were dissected, pooled together and conserved in $70 \%$ ethanol until further analysis. Samples ( $n=8$ per treatment) were analysed at the Department of Bacteria, Parasites and Fungi, Statens 
Serum Institut, Copenhagen, Denmark. DNA was extracted using a commercial kit (QIAampDNA Mini Kit; Qiagen) according to the manufacturer's instructions for tissue. For each batch of DNA extraction, a 'negative' control was included containing reagents only for downstream analysis. DNA was amplified using a 2-step polymerase chain reaction using custom primers $341 \mathrm{~F} / 806 \mathrm{R}$ (the forward primer had three additional nucleotides attached in the 5' end (ACTCCTAYGGGRBGCASCAG, 341F3) and the reverse primer had five additional nucleotides attached in the 5' end (AGCGTGGACTACNNGGGTATCTAAT, 806R5)) targeting the V3-V4 16S rRNA gene hypervariable regions, as well as three primer sets (G3F1/G3R1 (GCCAGCAGCCGCGGTAATTC / ACATTCTTGGCAAATGCTTTCGCAG), G4F3/G4R3 (CAGCCGCGGTAATTCCAGCTC / GGTGGTGCCCTTCCGTCAAT) and G6F1/G6R1 (TGGAGGGCAAGTCTGGTGCC / ACGGTATCTGATCGTCTTCGATCCC)) flanking the hypervariable regions V3-V4 of the $16 \mathrm{~S}$ rRNA gene, and amplicons were sequenced on a desktop sequencer (MiSeq; Illumina, Inc., San Diego, CA, USA) using the v2 reagent kit. Sequence data are available at the European Nucleotide Archive (accession number PRJEB36600). Sequences were mapped using BION, a k-mer-based mapping software described previously (in supplementary material of Ring et al., 2017). A unique taxon was defined by denotation to a specific evolutionary taxonomic group. Prior to community composition analysis, low abundance species were discarded. We retained taxa that were present in at least two study samples and whose average relative abundance across all samples was higher than $0.01 \%$. Additionally, we removed putative contaminants that were found to be present in the negative (no template) control included prior to sequencing analysis. To be retained, the estimated abundance of a taxon in each of at least three gut samples had to be at least 2-fold higher than in the negative control sample. These filtering steps eliminated 6 and 9 taxa, respectively, resulting in 31 identified taxa (of which 29 were identified down to species level and 2 down to the genus level). Data were not rarefied, as recommended by McMurdie and Holmes (2014).

\section{Community data and statistical analysis}

All tests and graphs evaluating in vitro inhibition and the effect of the bacterial strain on T. molitor development were carried out using IBM SPSS Version 24. Independentsamples t-tests were performed on the mean of the inhibition measurements for each pathogenic strain species. Average larval weight was determined by dividing the weight of all the larvae in a replicate by the number of larvae in that replicate. Differences were analysed using a generalised linear model with a Normal distribution, and multiple comparisons were adjusted using sequential Bonferroni. The effect of our treatments on the number of larvae and adults was estimated using a generalised linear model with a Poisson distribution, and multiple comparisons were adjusted using sequential Bonferroni. The effect of our treatment on the average number of eggs per female was assessed using a non-parametric Median test and Kruskal-Wallis test comparing medians and distributions respectively. For all tests a significance level was set at $P<0.05$.

Bacterial microbiota data were analysed in R v. 3.6.0. ( $\mathrm{R}$ core team, 2018). We quantified alpha diversity with two indices: (1) species richness (observed); and (2) Shannon diversity index, using 'phyloseq' package (McMurdie and Holmes, 2013). Differences in alpha diversity between treatments were assessed by ANOVA followed by post hoc analysis and false discovery rate (FDR)-correction for multiple testing. Data normality was examined prior to the analysis using the Shapiro-Wilk's test. Beta diversity was computed with the Bray-Curtis metric ('phyloseq' package). Differences in gut bacterial community composition (beta-diversity) among the three treatments were assessed by performing PERMANOVA on the Bray-Curtis distance matrix by using the 'adonis' command with 10,000 permutations in the 'vegan' package (Oksanen et al., 2019). Homogeneity of multivariate dispersion across treatments was verified using the 'betadisper' function in 'vegan'. A post hoc test using the 'pairwise.perm.manova' command within 'RVAideMemoire' package (Hervé, 2019) with 10,000 permutations and FDRcorrection for $P$-value was applied. To analyse differences in relative abundance of $P$. pentosaceus between treatments a Kruskal-Wallis non-parametric test followed by post hoc comparisons using the Dunn test (package 'FSA' (Ogle et al., 2018); function dunnTest) was applied and FDR-corrected $P$-values are reported.

\section{Results}

\section{In vitro antimicrobial activity}

Our screening of the antimicrobial activity of $P$. pentosaceus str. KVLB19-01 using the dual-overlay method revealed that activity of all six insect pathogenic bacteria was significantly inhibited by our strain (Figure 1). While the highest inhibition was observed against $S$. plymuthica (mean \pm s.e.: $12.00 \pm 0.29 ; \mathrm{t}=41.57 ; \mathrm{DF}=4 ; P<0.001$ ), the lowest was found against a species from the same genus, S. marscesens (mean \pm s.e.: $7.83 \pm 0.17 ; \mathrm{t}=47.00$; $\mathrm{DF}=4$; $P<0.001)$. The level of inhibition against all three tested B. thuringiensis strains was highly consistent (Bt1236 (mean \pm s.e.: $11.33 \pm 0.44 ; \mathrm{t}=25.70$; $\mathrm{DF}=4 ; P<0.001)$. Bt 34 (mean \pm s.e.: $11.50 \pm 0.00 ; \mathrm{t}=39.84 ; \mathrm{DF}=4 ; P<0.001)$. BtUK (mean \pm s.e.: $11.67 \pm 0.44 ; \mathrm{t}=26.46 ; \mathrm{DF}=4 ; P<0.001)$. $P$. aeruginosa also appeared inhibited by $P$. pentosaceus (mean \pm s.e.: $8.50 \pm 0.50 ; \mathrm{t}=17.00 ; \mathrm{DF}=4 ; P<0.001)$. 


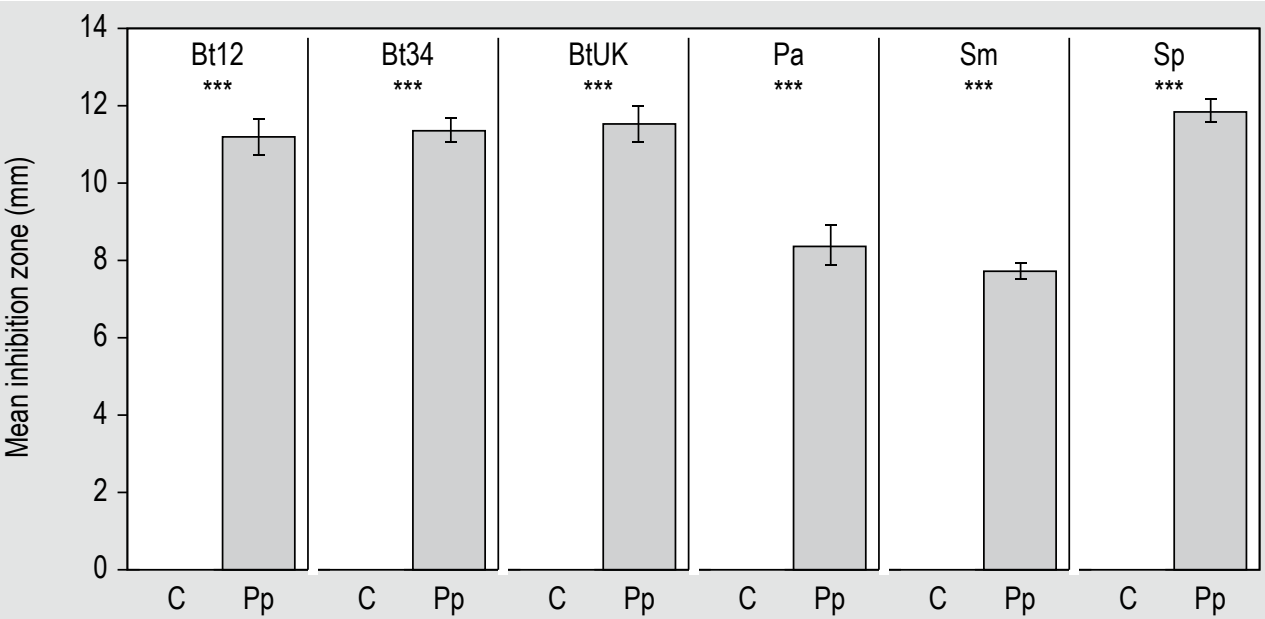

Figure 1. Dual overlay test of the Pediococcus pentosaceus $(\mathrm{Pp})$ isolate against six pathogenic bacteria: Bacillus thuringiensis (Bt12, Bt34 and BtUK), Pseudomonas aeruginosa (Pa), Serratia marcescens (Sm) and Serratia plymuthica (Sp). Growth inhibition scores (in $\mathrm{mm}$ ) for all three replicates are based on the average of two measurements, per replicate, of the radius of the inhibition zone. The final inhibition score (Mean) was defined as the average from the measurements taken from the replicates. A control (C) using sterile water instead of $P$. pentosaceus, confirmed growth potential with no inhibition, of each the pathogenic bacteria in the absence of $P$. pentosaceus. $P$. pentosaceus was able to inhibit the growth of all of the tested pathogens to some degree. Error bars represent \pm 1 S.E. from the mean. ${ }^{* * *}$ Significant at $P<0.001$.

\section{Characterisation of Pediococcus pentosaceus diet supplementation effects}

Between weeks 0 and 10, we measured the weight of the larvae in each of our replicates. We found overall significant differences in the effect of our treatment on larval weight $(\mathrm{F}=179.5 ; \mathrm{DF}=2 ; P<0.001)$. Post hoc tests adjusted with Bonferroni showed no significant differences among treatments at week 0 (expected) and week 4 . In all subsequent weeks, control (C) larvae weighed significantly less than both larvae for which feed was supplemented by live $(\mathrm{B})$ and autoclaved bacteria $(\mathrm{AB})(P<0.001)$. Larvae from the $B$ treatment group weighed significantly more than those from the $\mathrm{AB}$ treatment group at week $8(P=0.017)$. Larvae from the bacteria (live) supplemented diet group gained weight significantly faster than the larvae from our control group from between week 5 and week 7 and onwards and reached a harvest weight of $0.12 \mathrm{~g}$ approximately 1.5 weeks earlier than controls. By the time larvae in the control group reached a harvest weight, mealworms from the $B$ and $\mathrm{AB}$ treatments were 1.3- and 1.2-fold heavier, respectively (Figure 2).

We counted the number of surviving larvae at week 8 , the last week prior to the appearance of the first pupae, as well as the cumulated number of intact adults that had emerged up until week 20, the last week with any remaining larvae (Figures 3A and 3B). When analysing the effect of our treatments on the number of surviving larvae at week 8 we found significant overall differences of treatment $(\mathrm{F}=20.34 ; \mathrm{DF}=2 ; P<0.001)$. At that point, out of 100 larvae, an average of $57.88 \pm 1.47$ (mean \pm s.e.) larvae survived

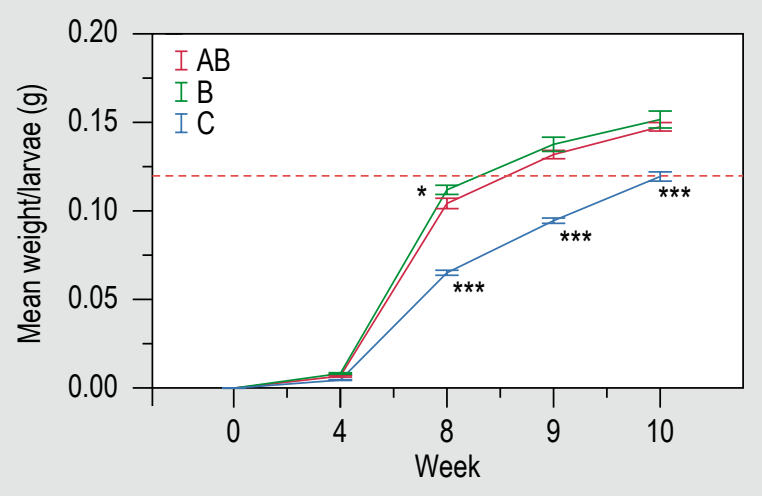

Figure 2. Average Tenebrio molitor larval weight over the course of 10 weeks. Horizontal reference line at $Y=0.12 \mathrm{~g}$ indicates the weight of the larvae at harvest. Blue: control (C); green: live bacteria (B); red: autoclaved bacteria (AB). We observed a significant effect of both $A B$ and $B$ treatments from week 8 and onwards and larvae in the $B$ treatment were significantly heavier than those of $A B$ treatment at week 8. Error bars represent \pm 1 S.E. from the mean. ${ }^{* * *}$ Significant at $P<0.001$; ${ }^{*}$ significant at $P<0.05$.

in the $\mathrm{C}$ group, $72.00 \pm 1.78$ in the $\mathrm{AB}$ treatment group, and $75.13 \pm 2.67$ larvae in the B group. Analysis of post hoc Bonferroni-corrected pairwise contrasts revealed significant differences between the $\mathrm{B}$ and $\mathrm{C}$ groups $(P<0.001)$ and between the $\mathrm{AB}$ and $\mathrm{C}$ groups $(P<0.001)$ with regard to the number of surviving larvae. There was no significant difference between groups $\mathrm{AB}$ and $\mathrm{B}(P=0.97)$. Furthermore, we found a significant effect of treatment on the total number of intact adults that had emerged until week 20 
$(\mathrm{F}=55.10 ; \mathrm{DF}=2 ; P<0.001)$. The average number of adults that emerged intact in the $C$ group was $23.88 \pm 2.97$ (mean \pm s.e.). In contrast, we finished the experiment with an average of $46.75 \pm 1.41$ adults in the $\mathrm{AB}$ group and $55.63 \pm 1.95$ adults in our B group. Analysis of post hoc Bonferroni-corrected pairwise contrasts revealed significant differences between the $\mathrm{B}$ and $\mathrm{C}$ groups $(P<0.001)$, between the $\mathrm{AB}$ and $\mathrm{C}$ groups $(P<0.001)$, and between the $\mathrm{AB}$ and $\mathrm{B}$ groups $(P=0.029)$ with regard to the final number of emerged, intact, adults.

We measured the reproductive fitness (fecundity) of adult female $T$. molitor by placing the first 20 adults to emerge from each replicate in separate cages and counting the amount of eggs laid per female over a period of 7 days. There was overall no significant effect of diet supplementation, neither with dead nor with live bacteria
$(\mathrm{F}=1.04 ; \mathrm{DF}=2 ; P=0.38)$. Females in the $\mathrm{B}$ group laid an average of $47.44 \pm 3.54$ (mean \pm s.e.) eggs compared with $38.01 \pm 5.36$ and $38.23 \pm 8.09$ in the $A B$ group and $C$ group, respectively (Figure $3 \mathrm{C}$ ). Furthermore, it is worth noting that the first oviposition trials in the $\mathrm{B}$ and $\mathrm{AB}$ groups began at least 17 days before those of the $C$ group due to a faster development in the former groups. Three of the replicates in our control group did not reach 20 adults over the period of our study and therefore could not be counted.

\section{Bacterial microbiota sequencing and analysis}

We used 16S rRNA gene sequencing to compare the gut microbial community of $T$. molitor individuals maintained on three different diets (i.e. control diet, $\mathrm{C}$; diet supplemented with live $P$. pentosaceus, $\mathrm{B}$; and diet
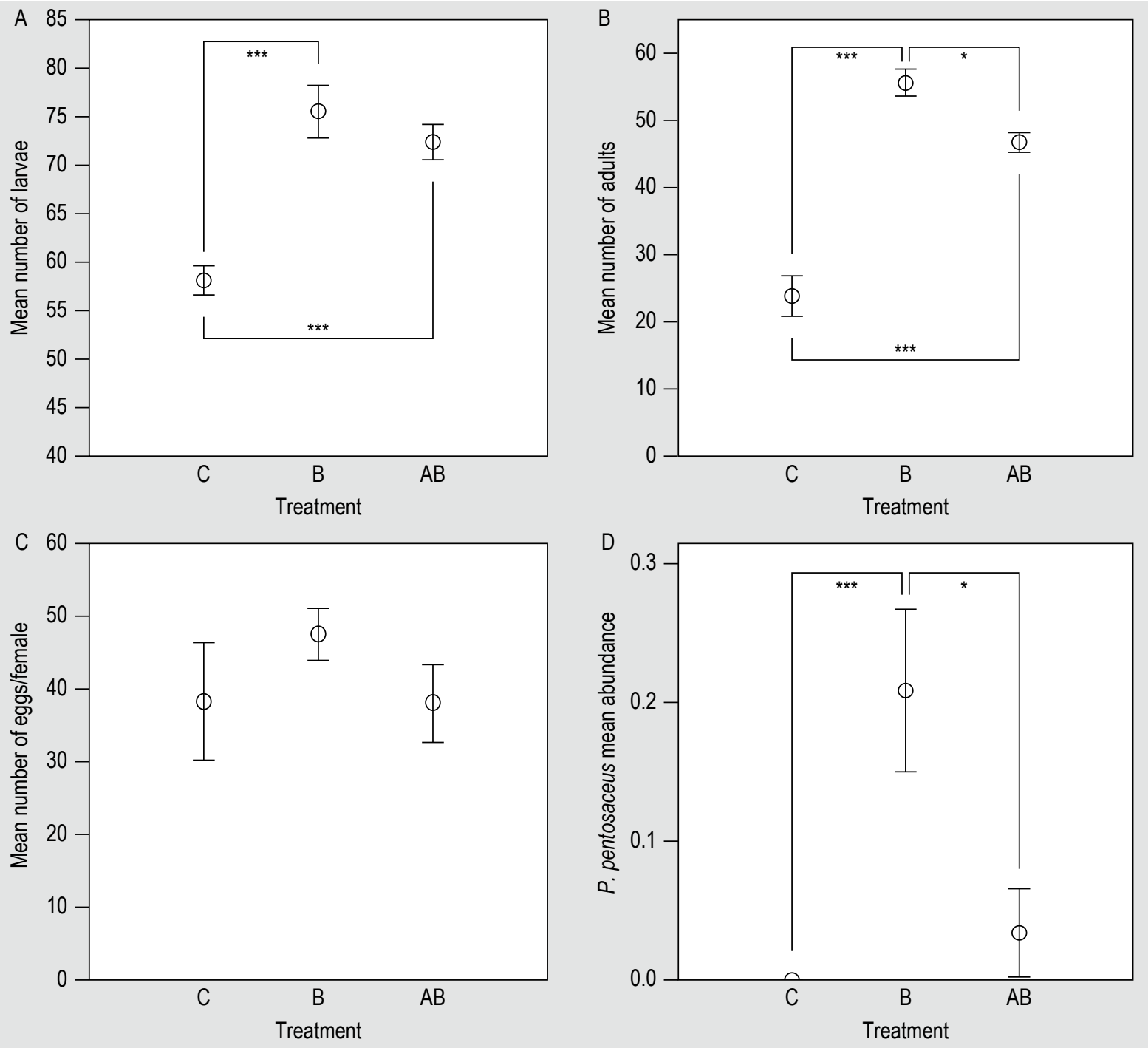

Figure 3. (A) average number of Tenebrio molitor larvae at week 8; (B) average number of adults at week 20; (C) average number of eggs per female in groups of 20 adults; (D) relative abundance of Pediococcus pentosaceus bacteria in the three treatments. Control (C); bacteria treatment (B); autoclaved bacteria treatment (AB). Error bars represent \pm 1 S.E. from the mean. ${ }^{* *}$ Significant at $P<0.001$; ${ }^{*}$ significant at $P<0.05$. 
supplemented with autoclaved $P$. pentosaceus, AB). We identified 31 unique taxa, and the main phyla identified were Firmicutes and Proteobacteria. The relative abundance of $P$. pentosaceus was significantly associated with treatment group (Kruskal-Wallis rank sum test, chi squared $=16.48$, $\mathrm{DF}=2, P<0.001$ ), being significantly higher in the $\mathrm{B}$ group that received the enriched diet compared with the $C$ group $(P<0.001)$, but also compared with the $\mathrm{AB}$ group $(P<0.004)$ (Figure 3D).

Moreover, the microbial profile of $\mathrm{C}$ group T. molitor samples was dominated by Enterobacteriales (relative abundance \pm s.e.: $0.42 \pm 0.14$ ) followed by Bacillales (relative abundance \pm s.e.: $0.24 \pm 0.11$ ), while in the B group, the community was strongly dominated by Lactobacillales (relative abundance \pm s.e.: $0.53 \pm 0.11$ ) followed also by Bacillales (relative abundance \pm s.e.: 0.22 \pm 0.09 ). In the $A B$ group, the community was dominated by Bacillales (relative abundance \pm s.e.: $0.46 \pm 0.11$ ) with Lactobacillales coming second (relative abundance \pm s.e.: 0.29 \pm 0.12 ) (Figure 4).

However, the gut microbial alpha diversity did not differ significantly between the three treatments (Observed: $\mathrm{F}_{(2,21)}=0.208, P=0.814$; Shannon: $\mathrm{F}_{(2,21)}=2.923, P=0.0759$; Figure 5). In addition, while PERMANOVA analysis using Bray-Curtis distances revealed a statistically significant difference in community composition with treatment explaining $13 \%$ of the variance $(P=0.03)$, pairwise comparisons did not show any significant differences between the three groups ( $C$ vs $A B: P=0.149 ; C$ vs $B$ : $P=0.076 ; \mathrm{AB}$ vs $\mathrm{B}: P=0.149$; Figure 6 ).

\section{Discussion}

The mass production of edible insects is gathering pace. Production facilities are rearing insects in greater numbers and densities than ever before. Preventing epidemics in the production and preparing for emerging diseases is becoming more important to protect this growing industry. Here we provide the first example of a host-associated probiotic bacterium, P. pentosaceus, isolated from the guts of T. molitor larvae, promoting larval growth and survival. We also found evidence of in vitro inhibition of bacterial pathogens by this bacterium. P. pentosaceus was able to significantly inhibit the growth of three $B$. thuringiensis strains. Bacillus thuringiensis is the most common bio pesticide in the world (Ibrahim et al., 2010). The implications of our strain being able to inhibit the growth of $B$. thuringiensis consistently could have wide-ranging applications and should be considered in the context of antibiotic resistance observed in agricultural pests. The Gram-negative bacteria, S. marcescens, S. plymuthica and $P$. aeruginosa were also inhibited by our potential probiotic. S. marcescens is the most common and pathogenic species of its genus and can also cause infections in humans (Yu, 1979). Although our strain showed the smallest inhibition zone against this pathogen, it was still able to supress its growth which could represent an advantage to insects in mass production and arguments for more research. Similarly, P. aeruginosa is a ubiquitous microorganism which has the ability to survive under a variety of environmental conditions. It not only causes disease in plants and animals, but also in humans, and may result in serious infections in immunocompromised patients with cancer and patients suffering from severe burns and cystic fibrosis (Wu et al., 2015). Overall, while our in vitro assay provides some interesting results, we still do not know how this observed inhibition translates to in vivo conditions. For example, it remains unclear whether the observed effect is strong enough to significantly affect a spreading infection within an insect population. Further experiments based on controlled infections in live mealworms are needed for confirmation. Moreover, the nature of the inhibition observed remains to be elucidated; however, based on recent studies, it appears that some strains of $P$. pentosaceus are able to produce bacteriocins, making them highly active against a number

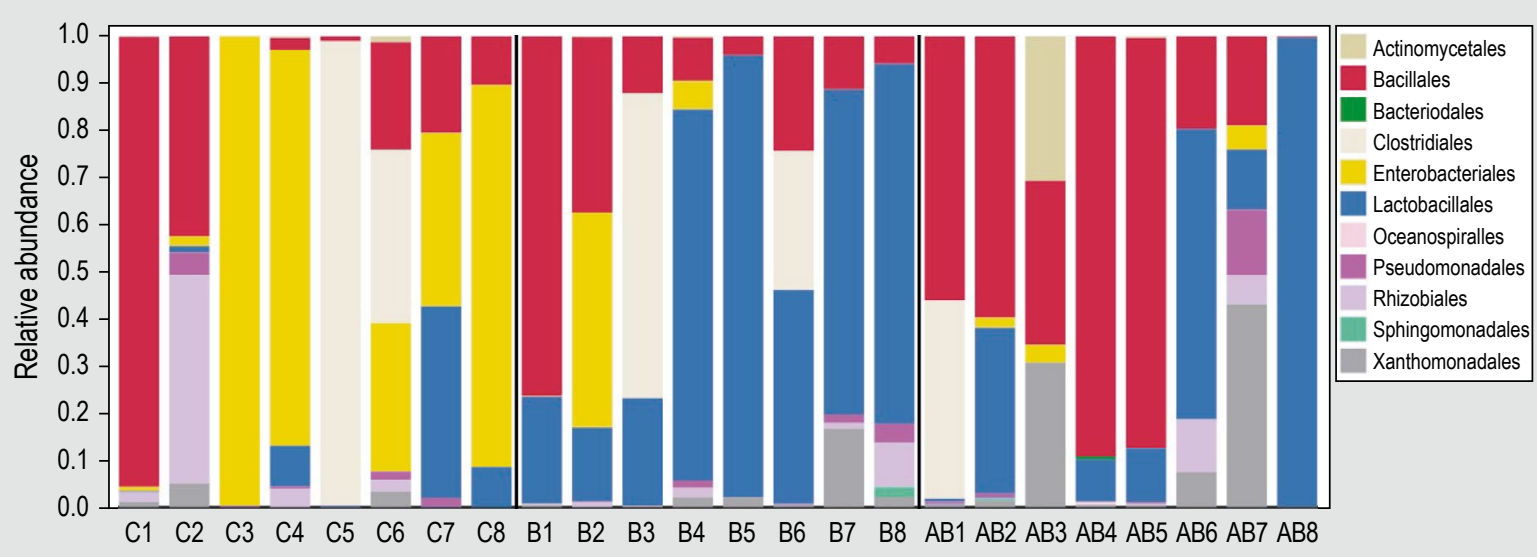

Figure 4. Relative abundance (from 0 to 1) of bacteria by order in control (C1-C8), bacteria (B1-B8), and autoclaved bacteria (AB1-AB8) treatments. 

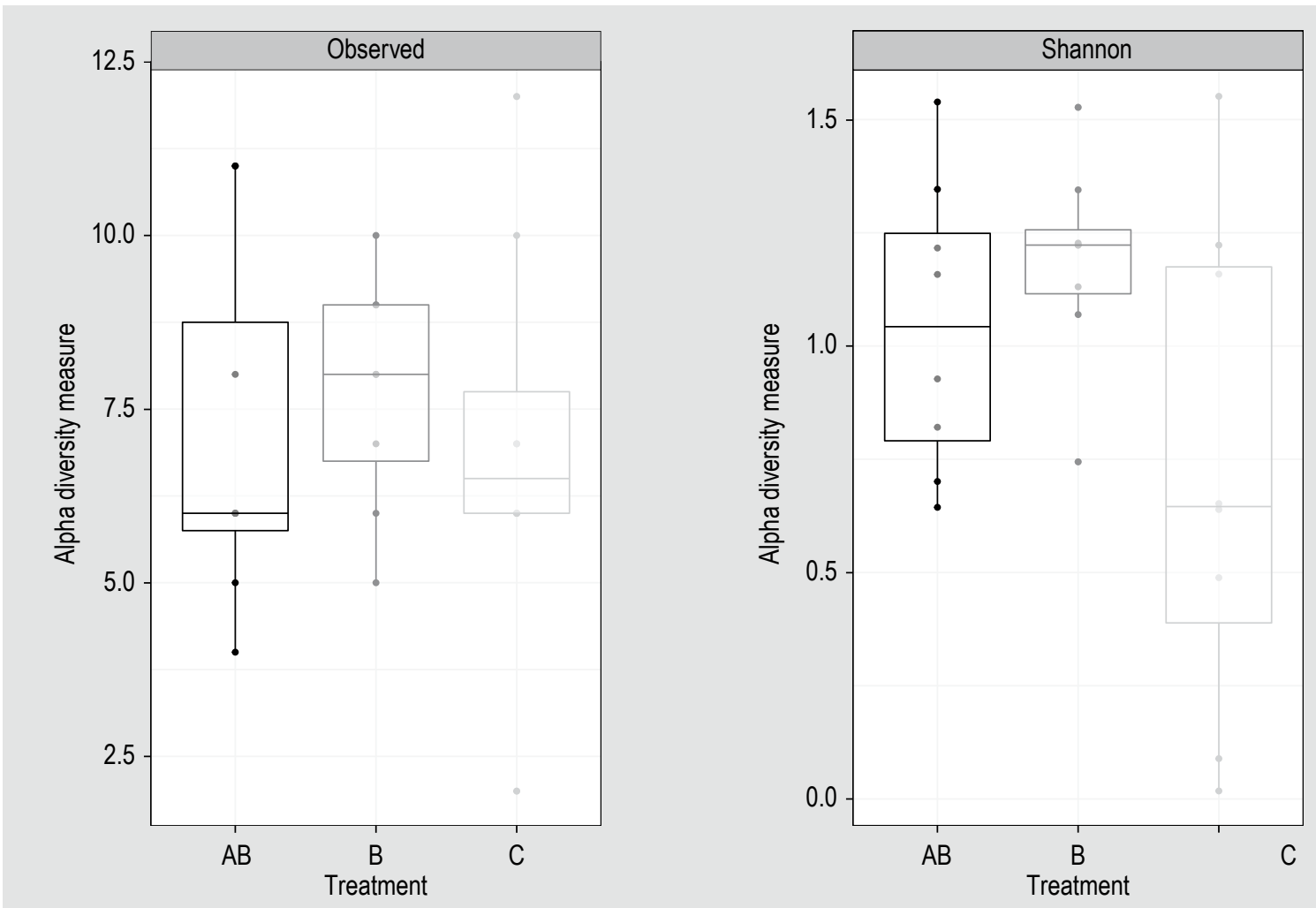

Figure 5. Alpha diversity indices (observed microbial richness and Shannon diversity). The boxplots represent diversity measures for the eight samples per treatment. Control (C); bacteria treatment (B); and autoclaved bacteria treatment (AB). The gut microbial alpha diversity did not differ significantly between the three treatments.

of bacterial and fungal pathogens (Cizeikiene et al., 2013; Gutiérrez-Cortés et al., 2018; Zommiti et al., 2018). Bacteriocins are proteinaceous antimicrobial substances produced by bacteria that can have a bactericidal mode of action (Klaenhammer, 1988). The precise mechanism by which our strain inhibited pathogen growth requires further study, but there appears to be research data pointing towards an effect of bacteriocins produced by Pediococcus sp. (Jamuna and Jeevaratnam, 2004; Papagianni and Anastasiadou, 2009; Vidhyasagar and Jeevaratnam, 2013). Those bacteriocins could also work in combination with other antagonistic metabolites such as hydrogen peroxide and organic acids, together affecting cellular signalling pathways and disrupting metabolic activities among other effects (Niederle et al., 2019). In our case, acid production by the P. pentosaceus strain could have lowered the $\mathrm{pH}$ of the MRS media to a level that would have inhibited the growth of some of the pathogenic strains used in the study. However, a study using other P. pentosaceus strains refuted this explanation as the sole reason for inhibitory effects on Gram-negative bacteria (Skyttä et al., 1993).

The fitness benefits associated with the presence of $P$. pentosaceus in the in vivo assays provide more evidence of its probiotic properties. Both our autoclaved and live bacteria reduced the mortality of the larvae and accelerated the rate of development. These results suggest that the autoclaved bacteria still contained properties that promoted larval growth. Research has shown that antimicrobial activity can be demonstrated in P. pentosaceus even after autoclaving cell-free-filtrate at $121^{\circ} \mathrm{C}$ for 15 minutes due to the presence of heat-resistant bacteriocins (Nghe and Nguyen, 2014). In that study the heat-resistant bacteriocins were not identified, however, it is known that one group of bacteriocins produced by Pediococcus spp. including P. pentosaceus, the pediocins can maintain their bactericidal action even after sterilisation conditions (Papagianni and Anastasiadou, 2009). Pediocins' maximum production is dependent on cultivation conditions (i.e. nutrition, $\mathrm{pH}$, temperature, aeration levels) that are species and/or strain dependant, reaching a peak at the late exponential phase of the culture (Nghe and Nguyen, 2014; Papagianni and Anastasiadou, 2009). For P. pentosaceus specifically, growth temperatures between 30 to $37^{\circ} \mathrm{C}$ in semi-aerobic conditions have been shown to result in optimum pediocin production depending on the strain (Papagianni and Anastasiadou, 2009). Thus, optimisation of growth culture conditions and harvesting time for $P$. pentosaceus could enhance pediocin production and thus bactericidal properties, paving the way for applications without the need for live cultures. Some research also suggests that specific strains of $P$. pentosaceus could stimulate the immune system and enhance the 


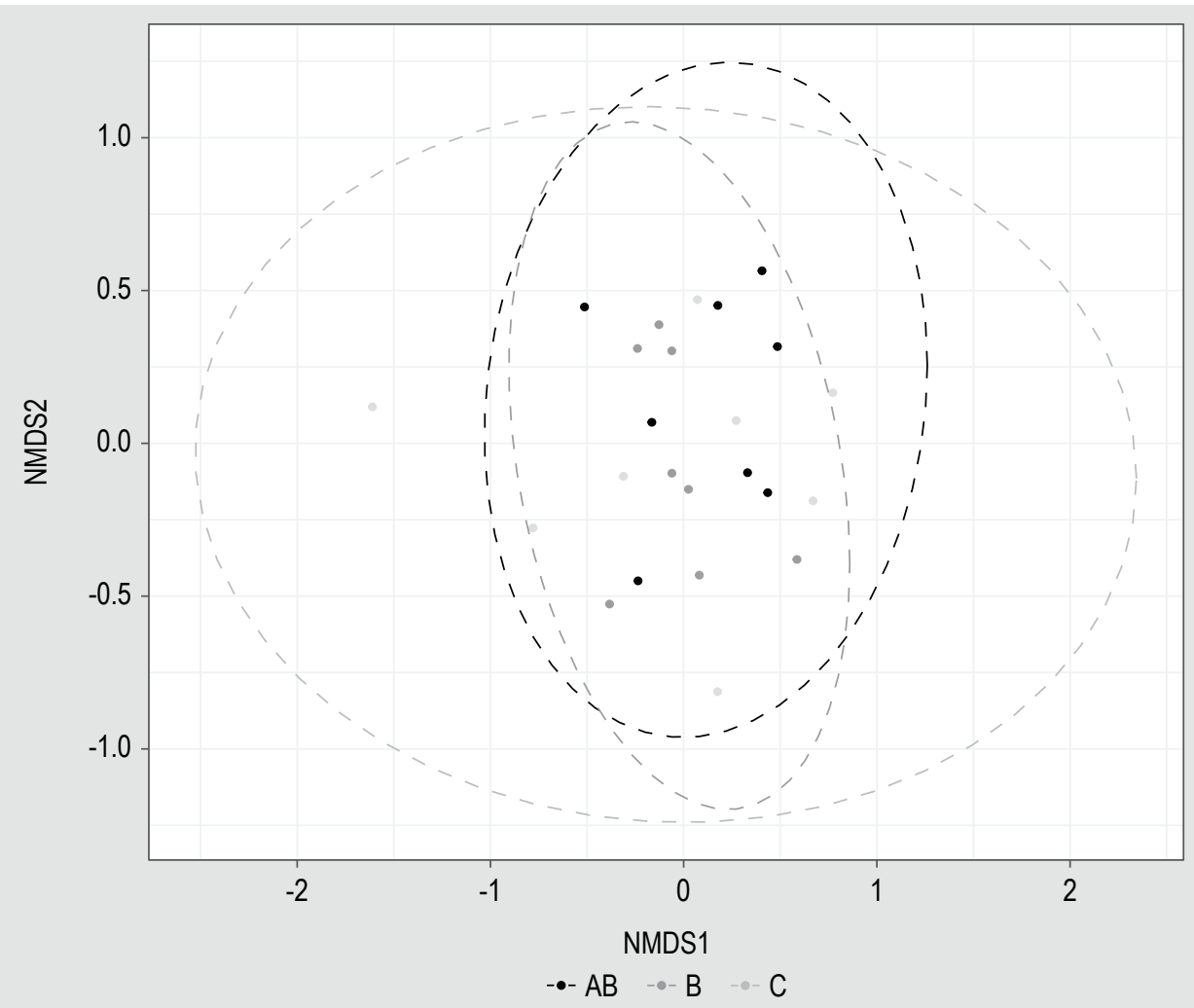

Figure 6. Beta diversity based on nonmetric multidimensional scaling (NMDS) ordination based on Bray-Curtis dissimilarity index. Control (C); bacteria treatment (B); and autoclaved bacteria treatment (AB). PERMANOVA analysis using Bray-Curtis distances revealed a statistically significant difference in community composition with treatment explaining $13 \%$ of the variance, pairwise comparisons did not show significant differences between the three groups.

intestinal epithelial barrier function (Zommiti et al., 2018). Other enzymes produced by $P$. pentosaceus could also have made the dry feed more palatable and enhance feeding (Juega et al., 2014). More research is needed to evaluate whether additional compounds from the bacteria, made readily available as a powder to young larvae, could have facilitated growth and survival. However, live bacteria do appear to provide additional protection as suggested by the significant increase in the number of intact adults to emerge in the live bacteria treatment group compared with both the control and autoclaved bacteria treatment groups.

Our analysis of the gut bacterial composition of T. molitor at harvest suggests that the insect consumed the bacteria and that the bacteria were likely to have colonised the guts in the live bacteria treatment as opposed to the autoclaved bacteria treatment. The relative abundance of $P$. pentosaceus in the guts of the larvae could also have promoted the growth of other species from the Lactobacillales. As Lactobacillales was also found to be the second most dominant order in the autoclaved group despite the low abundance of $P$. pentosaceus, any possible interplay between $P$. pentosaceus and other Lactobacilles species could potentially involve a combination of complex mechanisms that require living bacteria and/or their secreted molecules and needs further investigation.

Irrespectively of the mechanism, the predominance of $P$. pentosaceus and other Lactobacillales in the guts of the larvae could have a beneficial impact on the health of the next level consumer if left unprocessed, although many additional characterisation tests would need to be performed. Our taxonomic assignment results, using 16S sequencing, targeted the variable regions V3-V4 of the $16 \mathrm{~s}$ rRNA gene. Thus, taxonomic profiling and accuracy is based only on partial coverage of a single marker gene (i.e. 16s rRNA) and depends on comparison to previously classified $16 \mathrm{~s}$ reference databases. Whole genome shotgun metagenomic approaches that target all genomic DNA within a sample providing a much higher taxonomic resolution and accuracy, should be considered in future studies (Brumfield et al., 2020). Nonetheless, the 33 unique taxa identified here along with the identification of Firmicutes and Proteobacteria as the main phyla, are consistent with previous reports (Przemieniecki et al., 2020; Urbanek et al., 2020; Wynants et al., 2017). Other studies also observed a high abundance of bacteria from the Tenericutes phylum (especially Spiroplasma spp.), however, those were undetected in our samples (Jung et 
al., 2014; Wang and Zhang, 2015). While we observed changes in the relative abundance of bacterial orders in the larvae, these did not translate into significant changes in bacterial richness or diversity indices. The relatively high variability of individual gut bacterial composition could account for this lack of statistical significance. The source population for our experiment was part of a healthy small pilot production of T. molitor in Denmark, however, we were unable to assert the absolute absence of parasites and pathogens or a homogenous gut microbiota prior to our experiment. This effect was minimised by starting the experiment within $24 \mathrm{~h}$ of hatching and giving the larvae the same diet as previous generations. Similarly, we cannot rule out that some of the larvae carried some P. pentosaceus before the assay which could explain their presence in both control and autoclaved bacteria samples. The fact that the larvae were administered their treatment at week 4 , but guts were dissected between week 8 and 10 , could also mean that the effects of the bacteria on the gut bacterial microbiota were dissipating. Overall, providing protection to the insects in production is an important aspect deserving further study. The accelerated rise in the mass production of insects is forcing researchers and industry alike to consider insect health in all its aspects. The increase in antibiotic use observed among all groups of animals, from humans to honeybees (Tian et al., 2012) has forced us to rethink their use and to develop other innovative ways to manage pathogens in populations. While the scientific community focuses on its efforts on developing protocols for the identification and management of emerging pathogenic threats, there remains potential for the protection of insects and their consumers using dietary supplementation.

\section{Conflict of interest}

The authors declare that the research was conducted in the absence of any commercial or financial relationships that could be construed as a potential conflict of interest.

\section{Acknowledgements}

The work presented was part of the inVALUABLE project funded by Innovation Fund Denmark under the Grand Solution program (grant no. 6150-00011B).

\section{Data availability}

The datasets generated for this study can be found in the European Nucleotide Archive, PRJEB36600, ena-STUDYSTATENS SERUM INSTITUT-05-02-2020-15:22:10:169-18.

\section{References}

Bhattacharya, A.K., Ameel, J.J. and Waldbauer, G.P., 1970. A method for sexing living pupal and adult yellow mealworms. Annals of the Entomological Society of America 63(6): 1783-1783.

Bermudez-Brito, M., Plaza-Díaz, J., Muñoz-Quezada, S., GómezLlorente, C. and Gil, A., 2012. Probiotic mechanisms of action. Annals of Nutrition and Metabolism 61(2): 160-174.

Brumfield, K.D., Huq, A., Colwell, R.R., Olds, J.L. and Leddy, M.B., 2020. Microbial resolution of whole genome shotgun and $16 \mathrm{~S}$ amplicon metagenomic sequencing using publicly available NEON data. PLoS ONE 15(2): e0228899. https://doi.org/10.1371/journal. pone.0228899.

Carlsson-Kanyama, A., 1998. Climate change and dietary choices how can emissions of greenhouse gases from food consumption be reduced? Food Policy 23(3): 277-293.

Cizeikiene, D., Juodeikiene, G., Paskevicius, A. and Bartkiene, E., 2013. Antimicrobial activity of lactic acid bacteria against pathogenic and spoilage microorganism isolated from food and their control in wheat bread. Food Control 31(2): 539-545.

Eilenberg, J., Vlak, J.M., Nielsen-LeRoux, C., Cappellozza, S. and Jensen, A.B., 2015. Diseases in insects produced for food and feed. Journal of Insects as Food and Feed 1(2): 87-102.

Foley, J.A., Ramankutty, N., Brauman, K.A., Cassidy, E.S., Gerber, J.S., Johnston, M., Mueller, N.D., O'Connell, C., Ray, D.K., West, P.C., Balzer, C., Bennett, E.M., Carpenter, S.R., Hill, J.C. Monfreda, C., Polasky, S., Rockstrom, J., Sheehan, J., Siebert, S., Tilman, D. and Zaks, D.P.M., 2011. Solutions for a cultivated planet. Nature 478(7369): 337-342.

Ghaly, A.E. and Alkoaik, F., 2009. The yellow mealworm as a novel source of protein. American Journal of Agricultural and Biological Sciences 4(4): 319-331.

Grau, T., Vilcinskas, A. and Joop, G., 2017b. Probiotic Enterococcus mundtii isolate protects the model insect Tribolium castaneum against Bacillus thuringiensis. Frontiers in Microbiology 8: 1261.

Grau, T., Vilcinskas, A. and Joop, G., 2017a. Sustainable farming of the mealworm Tenebrio molitor for the production of food and feed. Zeitschrift für Naturforschung C72: 337-349.

Gutiérrez-Cortés, C., Suarez, H., Buitrago, G., Nero, L.A. and Todorov, S.D., 2018. Enhanced bacteriocin production by Pediococcus pentosaceus 147 in co-culture with Lactobacillus plantarum LE27 on cheese whey broth. Frontiers in Microbiology 9: 2952.

Hervé, M. and Hervé, M.M., 2019. Package 'RVAideMemoire'. Testing and plotting procedures for biostatistics. R-Project, Vienna, Austria. Available at: https://cran.r-project.org/web/packages/ RVAideMemoire/index.html

Hill, C., Guarner, F., Reid, G., Gibson, G.R., Merenstein, D.J., Pot, B., Morelli, L., Canani, R.B., Flint, H.J., Salminen, S., Calder, P.C. and Sanders, M.E., 2014. Expert consensus document: the International Scientific Association for Probiotics and Prebiotics consensus statement on the scope and appropriate use of the term probiotic. Nature Reviews Gastroenterology \& Hepatology 11(8): 506-514.

Holzapfel, W.H. and Schillinger, U., 2002. Introduction to pre-and probiotics. Food Research International 35(2-3): 109-116. 
Ibrahim, M.A., Griko, N., Junker, M. and Bulla, L.A., 2010. Bacillus thuringiensis: a genomics and proteomics perspective. Bioengineered Bugs 1(1): 31-50. https://doi.org/10.4161/bbug.1.1.10519

Islam, M.M. and Yang, C.-J., 2016. Efficacy of mealworm and super mealworm larvae probiotics as an alternative to antibiotics challenged orally with Salmonella and E. coli infection in broiler chicks. Poultry Science 96(1): 27-34.

Jamuna, M. and Jeevaratnam, K., 2004. Isolation and partial characterization of bacteriocins from Pediococcus species. Applied Microbiology and Biotechnology 65(4): 433-439.

Juega, M., Costantini, A., Bonello, F., Cravero, M.C., MartinezRodriguez, A.J., Carrascosa, A.V. and Garcia-Moruno, E., 2014. Effect of malolactic fermentation by Pediococcus damnosus on the composition and sensory profile of Albariño and Caiño white wines. Journal of Applied Microbiology 116(3): 586-595.

Jung, J., Heo, A., Park, Y. W., Kim, Y. J., Koh, H. and Park, W., 2014. Gut microbiota of Tenebrio molitor and their response to environmental change. Journal of Microbiology and Biotechnology 24(7): 888-897.

Klaenhammer, T.R., 1988. Bacteriocins of lactic acid bacteria. Biochimie 70(3): 337-349.

Lecocq, A., Jensen, A.B. and Eilenberg, J., 2019. Diseases of insects in European production systems. Berliner und Muenchener Tieräerztliche Wochenschrift 132(5-6): 245-250. https://doi. org/10.2376/0005-9366-18061.

Lilly, D.M. and Stillwell, R.H., 1965. Probiotics: growth-promoting factors produced by microorganisms. Science 147(3659): 747-748.

Magnusson, J. and Schnürer, J., 2001. Lactobacillus coryniformis subsp. coryniformis strain Si3 produces a broad-spectrum proteinaceous antifungal compound. Applied and Environmental Microbiology 67(1): 1-5.

McMurdie, P.J. and Holmes, S., 2013. Phyloseq: an R package for reproducible interactive analysis and graphics of microbiome census data. PLoS ONE 8: e61217. https://doi.org/10.1371/journal. pone.0061217

McMurdie, P.J. and Holmes, S., 2014. Waste not, want not: why rarefying microbiome data is inadmissible. PLoS Computational Biology 10(4): e1003531. https://doi.org/10.1371/journal. pcbi.1003531.

Nghe, D. and Nguyen, T., 2014. Characterization of antimicrobial activities of Pediococcus pentosaceus Vtcc-B-601. Journal of Applied Pharmaceutical Science 4(5): 61.

Niederle, M.V., Bosch, J., Ale, C.E., Nader-Macías, M.E., Ficoseco, C.A., Toledo, L.F., Valenzuela-Sánchez, A., Soto-Azat, C. and Pasteris, S.E., 2019. Skin-associated lactic acid bacteria from North American bullfrogs as potential control agents of Batrachochytrium dendrobatidis. PLoS ONE 14(9): e223020. https://doi.org/10.1371/ journal.pone.0223020.

Oelschlaeger, T.A., 2010. Mechanisms of probiotic actions - a review. International Journal of Medical Microbiology 300(1): 57-62.

Ogle, D.H., Wheeler, P. and Dinno, A., 2018. FSA: fisheries stock analysis. R package version 0.8. 20. Available at: https://github. com/droglenc/FSA

Oksanen, J., 2019. Vegan: community ecology package. R package. Available at: https://CRAN.R-project.org/package=vegan
Papagianni, M. and Anastasiadou, S., 2009. Pediocins: the bacteriocins of Pediococci. Sources, production, properties and applications. Microbial Cell Factories 8(1): 3.

Pelletier, N. and Tyedmers, P., 2010. Forecasting potential global environmental costs of livestock production 2000-2050. Proceedings of the National Academy of Sciences of the United States of America 107(43): 18371-18374.

Przemieniecki, S.W., Kosewska, A., Ciesielski, S. and Kosewska, O., 2020. Changes in the gut microbiome and enzymatic profile of Tenebrio molitor larvae biodegrading cellulose, polyethylene and polystyrene waste. Environmental Pollution 256: 113265.

$\mathrm{R}$ Core Team, 2018. $\mathrm{R}$ a language and environment for statistical computing. R Foundation for Statistical Computing, Vienna, Austria.

Ring, H.C., Thorsen, J., Saunte, D.M., Lilje, B., Bay, J., Riis, P.T., Larsen, N. andersen, L.O., Nielsen, H.V., Miller, I.M., Bjarnsholt, T., Fuursted, K. and Jemec, G.B., 2017. The follicular skin microbiome in patients with hidradenitis suppurativa and healthy controls. JAMA Dermatology 153: 897-905.

Skyttä, E., Haikara, A. and Mattila-Sandholm, T., 1993. Production and characterization of antibacterial compounds produced by Pediococcus damnosus and Pediococcus pentosaceus. Journal of Applied Bacteriology 74(2): 134-142.

Stoops, J., Crauwels, S., Waud, M., Claes, J., Lievens, B. and Van Campenhout, L., 2016. Microbial community assessment of mealworm larvae (Tenebrio molitor) and grasshoppers (Locusta migratoria migratorioides) sold for human consumption. Food Microbiology 53: 122-127.

Tian, B., Fadhil, N.H., Powell, J.E., Kwong, W.K. and Moran, N.A., 2012. Long-term exposure to antibiotics has caused accumulation of resistance determinants in the gut microbiota of honeybees. MBio 3(6): e00377-12. https://doi.org/10.1128/mBio.00377-12

Urbanek, A.K., Rybak, J., Wróbel, M., Leluk, K. and Mirończuk, A.M., 2020. A comprehensive assessment of microbiome diversity in Tenebrio molitor fed with polystyrene waste. Environmental Pollution 262: 114281. https://doi.org/10.1016/j.envpol.2020.114281

Van Huis, A., 2013. Potential of insects as food and feed in assuring food security. Annual Review of Entomology 58: 563-583.

Vidhyasagar, V. and Jeevaratnam, K., 2013. Evaluation of Pediococcus pentosaceus strains isolated from Idly batter for probiotic properties in vitro. Journal of Functional Foods 5(1): 235-243.

Wang, Y. and Zhang, Y., 2015. Investigation of gut-associated bacteria in Tenebrio molitor (Coleoptera: Tenebrionidae) larvae using culturedependent and DGGE methods. Annals of the Entomological Society of America 108(5): 941-949.

Wu, W., Jin, Y., Bai, F. and Jin, S., 2015. Pseudomonas aeruginosa. In: Sails, A. (ed.) Molecular medical microbiology. Academic Press, New York NY, USA, pp. 753-767.

Wilson, K., 2001. Preparation of genomic DNA from bacteria. Current Protocols in Molecular Biology 56: 2.4.1-2.4.5. https:// doi.org/10.1002/0471142727.mb0204s56

Wynants, E., Crauwels, S., Lievens, B., Luca, S., Claes, J., Borremans, A., Bruyninckx, L. and Van Campenhout, L., 2017. Effect of post-harvest starvation and rinsing on the microbial numbers and the bacterial community composition of mealworm larvae (Tenebrio molitor). Innovative Food Science \& Emerging Technologies 42: 8-15. 


\section{A. Lecocq et al.}

Yen, A.L., 2009. Edible insects: traditional knowledge or western phobia? Entomological Research 39(5): 289-298.

Yu, V.L., 1979. Serratia marcescens: historical perspective and clinical review. New England Journal of Medicine 300(16): 887-893.
Zommiti, M., Bouffartigues, E., Maillot, O., Barreau, M., Szunerits, S., Sebei, K., Feuilloley, M., Connil, N. and Ferchichi, M., 2018. In vitro assessment of the probiotic properties and bacteriocinogenic potential of Pediococcus pentosaceus MZF16 isolated from artisanal Tunisian meat ‘Dried Ossban'. Frontiers in Microbiology 9: 2607. 